\title{
Almanac 2011: Acute Coronary Syndromes. The National Society Journals Present Selected Research that has Driven Recent Advances in Clinical Cardiology
}

\author{
Charles Knight, Adam D. Timmis \\ Barts and the London School of Medicine and Dentistry London Chest Hospital, London, United Kingdom
}

\section{REVIEW}

SUMMARY

This overview highlights some recent advances in the epidemiology, diagnosis, risk stratification and treatment of acute coronary syndromes. The sheer volume of new studies reflects the robust state of global cardiovascular research but the focus here is on findings that are of most interest to the practising cardiologist. Incidence and mortality rates for myocardial infarction are in decline, probably owing to a combination of lifestyle changes, particularly smoking cessation, and improved pharmacological and interventional treatment. Troponins remain central for diagnosis and new high-sensitivity assays are further lowering detection thresholds and improving outcomes. The incremental diagnostic value of other circulating biomarkers remains unclear and for risk stratification simple clinical algorithms such as the GRACE score have proved more useful. Primary PCI with minimal treatment delay is the most effective reperfusion strategy in ST elevation myocardial infarction (STEMI). Radial access is associated with less bleeding than with the femoral approach, but outcomes appear similar. Manual thrombectomy limits distal embolisation and infarct size while drug-eluting stents reduce the need for further revascularisation procedures. Non-culprit disease is best dealt with electively as a staged procedure after primary $\mathrm{PCl}$ has been completed. The development of antithrombotic and antiplatelet regimens for primary $\mathrm{PCl}$ continues to evolve, with new indications for fondaparinux and bivalirudin as well as small-molecule glycoprotein (GP)IIb/IIla inhibitors. If timely primary PCI is unavailable, fibrinolytic treatment remains an option but a strategy of early angiographic assessment is recommended for all patients. Non-ST segment elevation myocardial infarction (NSTEMI) is now the dominant phenotype and outcomes after the acute phase are significantly worse than for STEMI. Many patients with NSTEMI remain undertreated and there is a large body of recent work seeking to define the most effective antithrombotic and antiplatelet regimens for this group of patients. The benefits of early invasive treatment for most patients are not in dispute but optimal timing remains unresolved. Cardiac rehabilitation is recommended for all patients with acute myocardial infarction but take-up rates are disappointing. Home-based programmes are effective and may be more acceptable for many patients. Evidence for the benefits of lifestyle modification and pharmacotherapy for secondary prevention continues to accumulate but the argument for omega-3 fatty acid supplements is now hard to sustain following recent negative trials. Implantable cardioverter-defibrillators for patients with severe myocardial infarction protect against sudden death but for primary prevention should be based on left ventricular ejection fraction measurements late (around 40 days) after presentation, earlier deployment showing no mortality benefit.

Key words: acute coronary syndromes, advances in clinical cardiology.

\section{INCIDENCE AND MODE OF PRESENTATION}

Temporal trends for the global coronary epidemic vary by region but in most developed countries mortality is in decline. ${ }^{1}$ Lifestyle adjustments have contributed to this decline-most recently, the implementation of comprehensive smoke-free legislation in many countries that has already caused significant reductions in acute coronary events. ${ }^{2}$ Smoking, a potent thrombogenic stimulus, is a major determinant of STEMI ${ }^{3}$ and a recent analysis from Kaiser Permanente in California-where smoke-free legislation is strictly enforced-showed a $62 \%$ decline in STEMI between 1999 and 2008 while NSTEMI increased by $30 \% .{ }^{4}$ Overall, there was a $24 \%$ reduction in hospitalisations for acute coronary syndromes despite lowering of diagnostic thresholds by sensitive troponin biomarkers. $\frac{5}{T}$ This was accompanied by improvement in the age- and sex- adjusted 30-day mortality from $10.5 \%$ in 1999 to $7.8 \%$ in 2008 . Increasing rates of interventional management no doubt contributed to the improved outcomes but parallel increases in plaquestabilising treatment with high-dose statins must also have played a role ${ }^{6}$ because vulnerable thin-cap fibroatheromas, often remote from the infarct-related artery and unrelated 
to stenosis severity, are the sites at which recurrent plaque events usually occur. $\underline{78}$

\section{DIAGNOSIS}

Diagnostic definitions of acute coronary syndromes are internationally agreed based on troponin release and symptomatic, electrocardiographic, or functional criteria. ${ }^{9}$

\subsection{Troponins}

Demonstration of a changing troponin concentration in the first $24 \mathrm{~h}$ with at least one value above the decision limit is central to the diagnosis of acute myocardial infarction. Now available are high-sensitivity troponin assays permitting significant reductions in the threshold for detection. An early study evaluated four high-sensitivity assays in 718 patients with suspected acute coronary syndrome, $17 \%$ of whom had acute myocardial infarction. Diagnostic performance was excellent, the area under the receiver operator curves ranging from 0.95 to 0.96 compared with 0.90 for the standard assay. 10 The implications for cardiac outcomes and clinical management were assessed in a more recent study in which high-sensitivity troponin I was measured in 1038 patients with suspected acute coronary syndrome. .1 $^{11}$ Values below the previous limit of detection $(0.20 \mathrm{ng} /$ $\mathrm{ml}$ ) - conventionally considered 'normal' - showed graded association with death or non-fatal myocardial infarction, with rates of $7 \%$ and $39 \%$ for troponin concentrations of $<0.05 \mathrm{ng} / \mathrm{ml}$ and $0.05-0.19 \mathrm{ng} / \mathrm{ml}$, respectively. When the investigators lowered the diagnostic threshold to $0.05 \mathrm{ng} /$ $\mathrm{ml}$ in a further 1054 patients, communicating troponin values to clinicians, the risk of death and recurrent myocardial infarction in patients with troponin concentrations $0.05-0.19 \mathrm{ng} / \mathrm{ml}$ was reduced from $39 \%$ to $12 \%$. The investigators concluded that lowering the diagnostic threshold by clinical application of high-sensitivity troponin assay has the potential to identify many high-risk individuals with suspected acute coronary syndrome and produce major improvements in their prognosis.

\subsection{Other diagnostic biomarkers}

Studies evaluating new biomarkers for the early diagnosis of myocardial infarction have been the subject of a recent systematic review. $\frac{12}{}$ The quality of these studies has often been poor with only $16 \%$ providing any information about incremental value compared with other diagnostic data. Myoglobin, for example, appears to be useful to rule out myocardial infarction in the first $6 \mathrm{~h}$ but evidence that it adds value to clinical symptoms, ECG and troponin testing is limited. Of the new diagnostic biomarkers, ischaemia-modified albumin and heart-type fatty acid-binding protein (H-FABP) showed initial promise, but already a meta-analysis has concluded that H-FABP does not fulfil the requirements needed for early diagnosis when used as a stand-alone test and called for evidence that it adds to clinical evaluation and other diagnostic tests. $\underline{13}$

\subsection{Point-of-care diagnosis with a panel of biomarkers}

Whether biomarker panels have a specific role for early diagnosis of myocardial infarction in the emergency room has been evaluated in two recent studies, both using a pointof-care panel of troponin I, creatine kinase-MB (CK-MB) and myoglobin. RATPAC recruited 2243 patients with suspected myocardial infarction and randomised them to standard care or panel evaluation on admission to the emergency room and 90 min later..$^{14}$ Point-of-care panel evaluation was associated with a $32 \%$ rate of 'successful' (no re-attendance with major coronary events) discharge from the emergency room, compared with 13\% for standard care; hospital bed use was unaffected. However, a substudy to examine the diagnostic efficiency of the individual cardiac markers and their accuracy for the final diagnosis of acute myocardial infarction showed that point-of-care myoglobin and CK-MB did not provide further diagnostic information over that provided by troponin I for early diagnosis or exclusion of myocardial infarction. $\frac{15}{}$ ASPECT was an observational study of 3582 patients in which an accelerated diagnostic panel (ADP) of TIMI score, coupled with the point-of-care panel of biomarkers and ECG findings, identified 352 as low risk. ${ }^{16}$ Only three of these patients went on to experience a major adverse cardiac event, making the ADP a highly sensitive rule-out for myocardial infarction in low-risk patients, as reflected by a negative predictive value of $99.1 \%$. However, there was no control group in ASPECT, nor an analysis of the incremental value offered by individual components of the biomarker panel. Based on the RATPAC subgroup analysis, therefore, it seems clear that troponin remains the most useful biomarker for diagnosis of myocardial infarction in the emergency room and current evidence is insufficient to advocate biomarker panels for this purpose.

\subsection{Electrocardiogram}

Guideline recommendations are for urgent reperfusion therapy according to STEMI pathways in patients with suspected myocardial infarction presenting with left bundle branch block (LBBB). However, a retrospective analysis of 892 patients in a Mayo Clinic STEMI registry, found that of the 36 who presented with new LBBB, only 12 (33\%) had a final diagnosis of acute myocardial infarction. ${ }^{17}$ These data show that LBBB is of limited diagnostic utility in suspected myocardial infarction and provide a case for new diagnostic strategies in this high-risk group. Also at high risk are patients with acute myocardial infarction caused by proximal left anterior descending coronary artery (LAD) occlusion. A report that this may be associated with a distinct ECG pattern has now been confirmed in a series of 35 patients who underwent primary PCI of the LAD, all of whom showed ST-segment depression at the J-point with up-sloping ST segments and tall, symmetrical T-waves in the precordial leads of the 12-lead ECG. ${ }^{18} 19$ The authors recommend that this ECG pattern in patients presenting with suspected myocardial infarction should prompt triage for immediate reperfusion therapy.

\subsection{Imaging}

Echocardiography provides the most readily available imaging modality for acute phase diagnosis of myocardial infarction by identifying new left ventricular regional wall motion abnormality. A new diagnostic application for identifying those patients with NSTEMI who have complete coronary occlusions was recently described..$^{20}$ In such patients, circumferential strain measured within 1 h of admission was independently diagnostic, values $\geq 10 \%$ showing $90 \%$ sensitivity and $88 \%$ sensitivity for angiographic coronary 
occlusion. The authors suggest that strain measurements in the acute phase of NSTEMI might be used for triaging patients for immediate reperfusion therapy.

\subsection{Risk stratification}

The risk of death and other ischaemic events in patients with acute coronary syndromes varies considerably across diagnostic phenotypes. Objective criteria to quantify risk are now increasingly used to guide treatment and determine prognosis.

\section{CLINICAL FACTORS}

Clinical factors are used intuitively by clinicians. They recognise that risk increases with age and shows important gender differences-young women with STEMI, for example, having a 15-20\% higher mortality risk than men. ${ }^{21}$ ECG criteria ${ }^{22}$ and routine biochemistry are also used for risk stratification, outcomes worsening with admission hyperglycaemia and also it seems with admission hypoglycaemia. $\stackrel{2324}{ }$ Despite clinicians' reliance on clinical assessments of risk it is now clear that they often get it wrong and a recent study has shown little association with objective measures of risk using validated risk scores. $\underline{25}$

\subsection{Diagnostic biomarkers}

Increasing troponin release in NSTEMI is associated with a proportionate increase in the risk of lethal arrhythmias, cardiogenic shock, new heart failure and death. $\frac{26}{6}$ C-reactive protein, the most widely studied prognostic biomarker, is also moderately predictive of adverse outcomes in acute coronary syndromes, a recent meta-analysis reporting a pooled RR of 2.18 (1.77 to 2.68) for the top ( $>10 \mathrm{mg} / \mathrm{l})$ compared with the bottom $(\leq 3 \mathrm{mg} / \mathrm{l})$ category of values, ${ }^{27}$ Generally speaking, however, individual biomarkers have yet to find a useful clinical role-a recent 5-year follow-up of patients with NSTEMI included in FRISC II reporting that none of $\mathrm{N}$-terminal pro-brain natriuretic peptide (NT-proBNP), C-reactive protein, cardiac troponin I and estimated glomerular filtration rate provided incremental prognostic value to established risk indicators, except NT-proBNP for 6 -week outcomes..$^{28}$ Combining multiple biomarkers may improve predictive power for adverse outcomes but confirmation of incremental value over established risk scores is still awaited. ${ }^{29}$

\subsection{Risk scores}

Validated risk scores based on a range of readily available factors provide the most effective means of risk stratifying patients with acute coronary syndromes. The GRACE score is widely used and in a comparative validation study involving 100686 cases of acute coronary syndromes its discriminative performance in predicting mortality compared favourably with a range of other risk models including PURSUIT, GUSTO-1, GRACE, SRI and EMMACE. $\underline{.30}$ The GRACE score appears to have lost none of its clinical value with the availability of high-sensitivity cardiac troponin assays. In an international cohort of 370 patients with acute coronary syndromes, the area under the curve of the GRACE score was 0.87 and 0.88 for in-hospital and 1-year mortality, and addition of high-sensitivity cardiac troponin produced no improvement in the mortality prediction. .1

\subsection{Primary percutaneous coronary intervention}

The MINAP public report for England and Wales records that $70 \%$ of all patients with STEMI received reperfusion therapy in 2010/2011, of whom $81 \%$ received primary PCI. . $^{32}$ The drive towards primary PCI, based on evidence of a sustained mortality benefit compared with fibrinolysis, $\underline{33}$ has been underpinned by the establishment of regional networks that have defined local standards of care and provided infrastructure for staffing heart attack centres. $\underline{3435}$

Timely treatment is essential to maximise prognostic benefit, ${ }^{\frac{3637}{}}$ and important as it is to achieve door-to-balloon times within $90 \mathrm{~min}$, other intrinsic delays within the healthcare process also need consideration. Thus, a Danish registry analysis of 6209 patients with STEMI found that 'system delay' (time from first contact with the healthcare system to the initiation of reperfusion therapy) - as well as door-to-balloon time-was a key modifiable risk factor, with an HR for mortality during the next 3.4 years of 1.22 (95\% CI 1.15 to 1.29 ; $\mathrm{p}<0.001$ ) per $1 \mathrm{~h}$ increase in system delay. ${ }^{38}$ The findings emphasise the importance of minimising transfer times from non-PCI hospitals and introducing policies of prehospital diagnosis to permit direct delivery of patients with STEMI to interventional centres. Also important are strategies to reduce the time it takes people with chest pain to call the emergency services. Women take significantly longer than men but, despite a US campaign to increase women's awareness of their risk of heart disease, a recent study found it had no effect on the gender gap or the time it took women to call the emergency services. ${ }^{39}$

\subsection{Vascular access}

Primary PCI by radial rather than femoral access is the preferred approach for an increasing number of operators. ${ }^{40}$ Its main advantage appears to be a lower rate of bleeding complications - the randomised RIVAL trial of radial versus femoral access in 7021 patients with acute coronary syndromes reporting a trend towards lower bleeding rates at 30 days ( $0.7 \%$ vs $0.9 \%)$, associated with significantly lower rates of access-site complications, including large haematomas and pseudoaneurysms. $\underline{41}$ Findings were similar in a recent observational study of 1051 primary PCI cases with vascular complication rates of $0 \%$ and $1.9 \%$ for radial versus femoral access. $\stackrel{42}{ }$ However, RIVAL found no outcome advantage for radial access, and femoral access is still preferred by many operators ${ }^{43}$ because access is more predictable and procedure times may be shorter than with the radial approach. $\underline{445}$

\subsection{Stenting}

Concerns about stent thrombosis led to recommendations for bare metal stents in primary PCI but randomised trials have now confirmed important advantages for drugeluting stents. The HORIZONS-AMI 3-year results showed lower rates of target lesion revascularisation for the 2257 patients randomised to paclitaxel-eluting stents than for the 749 patients randomised to bare metal stents $(9.4 \%$ vs $15.1 \%)$. $\underline{46}$ There was no difference by stent type in rates of death, reinfarction, stroke or stent thrombosis. Drugeluting stents are, therefore, preferred in primary PCI but they commit the patient to a full 12 months of dual antiplatelet treatment and if urgent surgery is planned or there is a high risk of bleeding for other reasons bare metal 
stents should be chosen.

\section{CULPRIT LESION VERSUS MULTIVESSEL PCI}

The main purpose of primary PCI is to achieve reperfusion of jeopardised myocardium by reopening the culprit coronary artery. Whether it is safe or desirable to treat disease within non-culprit vessels during the primary PCI procedure or as a staged procedure afterwards has been the subject of recent investigation. A small randomised trial of 214 patients with multivessel disease found that adverse event rates during a mean follow-up of 2.5 years were higher with culprit PCI than with multivessel PCI whether performed during the primary PCI procedure or, better, as a staged procedure afterwards. ${ }^{47}$ This trial has now been included in a meta-analysis of four prospective and 14 retrospective studies involving 40280 patients, which came to a similar conclusion in showing that staged PCI was associated with lower mortality compared with culprit PCI. $\stackrel{48}{ }$ However, multivessel PCI during the primary procedure was associated with the highest mortality. A post hoc analysis of the HORIZONS-AMI trial also found that staged PCI was associated with lower 1-year mortality compared with culprit PCI (2.3\% vs 9.2\%). 9 These data, are consistent in showing that multivessel disease is best dealt with electively as a staged procedure after the primary PCI procedure has been completed.

\section{THROMBECTOMY}

Thrombotic coronary occlusion is the pathological event triggering STEMI and provides the logic for adjunctive thrombectomy during primary PCI. A variety of devices have been developed for this purpose but the simplest, manual thrombus aspiration, has emerged as the best, with evidence of better reperfusion during the acute phase of STEMI translating into a survival advantage at 1 year compared with conventional primary PCI. $\underline{51}$ MRI has confirmed that thrombus aspiration reduces microvascular obstruction during primary PCI and limits infarct size at 3 months. $\frac{52}{}$ A more recent analysis of pooled individual patient data from three randomised trials found that the trend for worsening myocardial reperfusion with time from admission to primary PCI was effectively abolished by thrombus aspiration, suggesting particular benefits in the event of procedural delay. $\frac{53}{}$ More complex thrombectomy devices are not recommended for use in STEMI. Thus assessments of infarct size reduction in two trials-JETSTENT comparing Angiojet rheolytic thrombectomy with primary direct stenting and PREPARE comparing simultaneous proximal embolic protection and manual thrombus aspiration with manual thrombus aspiration-showed no significant benefit of these device strategies. ${ }^{5455}$ Consistent with this is a meta-analysis of thrombectomy trials showing that the mortality benefit for patients randomised to thrombus extraction is confined to patients treated with manual thrombectomy. $\underline{56}$

\section{ANTIPLATELET STRATEGIES}

Current recommendations are for loading doses of aspirin and clopidogrel immediately before primary PCI followed by maintenance treatment. Adjunctive treatment with GPIIb/IIIa receptor blockers provides more intensive platelet inhibition in the acute phase. The main purpose of treatment is to enhance thrombus resolution and to prevent recurrent thrombotic events, particularly stent thrombosis in the 9-12 months it takes for drug-eluting struts to endothelialise (1-3 months for bare metal struts). Newer, drugs that block the ADP P2Y12 receptor more potently than clopidogrel are now available ${ }^{57}$ and have been evaluated in combination with aspirin in patients undergoing primary PCI. In the TRITON-TIMI 38 trial of dual antiplatelet treatment, prasugrel reduced the primary outcome of cardiovascular death, non-fatal myocardial infarction and non-fatal stroke compared with clopidogrel (6.5\% vs 9.5\%), but this was associated with a significantly greater risk of major bleeding, including fatal bleeding, raising important safety concerns. $\stackrel{58}{ }$ Ticagrelor has also been evaluated against clopidogrel in a substudy of the PLATO trial and like prasugrel it proved more effective in reducing the primary outcome of cardiovascular death, myocardial infarction or stroke, although the absolute difference was small (9.0\% vs 10.7\%). . 9 Strikingly, however, there appeared to be enhanced bleeding, and ticagrelor now has a guideline recommendation for use in primary PCI, although its final place in the therapeutic arsenal must await costeffectiveness and long-term safety studies.

Abciximab, given intravenously, has been the most widely used GPIIb/IIIa receptor blocker in patients with STEMI undergoing primary PCI. Benefits appear to be inversely related to inflammatory burden ${ }^{60}$ and may be enhanced by intracoronary administration, a recent meta-analysis reporting improved clinical outcomes by this route. ${ }^{61}$ However, abciximab is expensive and there are now studies confirming non-inferiority of 'small-molecule' GPIIb/IIIa receptor blockers. Thus, investigators using the Swedish Coronary Angiography and Angioplasty Registry compared 2355 primary PCI patients who received eptifibatide with 9124 who received abciximab and found similar rates of death or myocardial infarction during 1-year follow-up (15.0\% vs $15.7 \%) .62$ In a smaller study, 427 patients randomised either to eptifibatide or abciximab showed comparable rates of complete ST-segment resolution 60 min after primary PCI (62.6\% vs $56.3 \%)$ with no significant differences between cardiovascular outcomes. ${ }^{63}$ In the On-TIME 2 trial, another small molecule compound, tirofiban, in combination with aspirin and clopidogrel, provided more effective platelet inhibition than aspirin and clopidogrel alone in patients undergoing primary PCI. The degree of platelet inhibition showed significant relationship with major adverse cardiac events, including stent thrombosis. $\underline{64}$ These findings have yet to penetrate international guidelines but many centres are now switching from abciximab to small-molecule compounds to reduce pharmacological costs. 


\section{OTHER ANTITHROMBOTIC DRUGS 7.1. Fondaparinux}

Intravenous heparin during primary PCI further enhances thrombus resolution during primary PCI but ongoing treatment with low molecular weight heparin has now given way to fondaparinux, a synthetic factor Xa inhibitor. A recent individual patient-level combined analysis of 26512 patients from the OASIS 5 and 6 trials who were randomised to fondaparinux $2.5 \mathrm{mg}$ daily or a heparinbased strategy has resolved uncertainty about the clinical value of fondaparinux in patients undergoing primary PCI by showing a better net clinical composite of death, myocardial infarction, stroke, or major bleeding (10.8\% vs 9.4\%; $\mathrm{HR}=0.87 ; \mathrm{p}=0.008)$ in the subset of 19085 patients treated invasively. $\frac{65}{}$ A similar benefit was found in patients treated conservatively. Fondaparinux is now widely used in preference to heparin in acute coronary syndromes.

\subsection{Bivalirudin}

Bivalirudin is a direct thrombin inhibitor that showed superiority to a combined regimen of heparin plus a GPIIb/ IIIa inhibitor in HORIZONS-AMI, largely owing to a lower rate of major bleeding (4.9\% vs $8.3 \%$ ). $\underline{6}$ All-cause mortality at 30 days was also lower in the bivalirudin group, with persistent benefit after 3 years (5.9\% vs 7.7\%), assuring a guideline recommendation for bivalirudin in primary PCI. ${ }^{46}$ It should be noted, however, that femoral artery access was used in $94.1 \%$ of the HORIZONS-AMI population and whether the reduction in bleeding with bivalirudin applies equally to centres where radial access is the preferred approach is not known.

\subsection{Fibrinolytic treatment}

Evidence that fibrinolysis is less effective than primary PCI in the emergency management of STEMI, has now been reinforced by evidence of reduced cost-effectiveness, ${ }^{67}$ yet a significant minority of patients in England and Wales continue to be treated with it. $\frac{32}{}$ This may be justified if fibrinolysis can be delivered within 30 min after presentation when primary PCI is not immediately available, because treatment delays by either modality are associated with substantial increases in mortality. ${ }^{36}$ This has provided justification for programmes of pre-hospital thrombolysis, particularly in rural regions where transport times are prolonged, but enthusiasm for this approach may now be diminished by evidence from the MINAP registry showing higher rates of reinfarction compared with in-hospital thrombolytic treatment for patients with STEMI. 68 The difference in reinfarction rates was only significant for tenecteplase $(9.6 \%$ vs $6.4 \%)$, not reteplase, and was particularly marked when transport times exceeded $30 \mathrm{~min}$. It was attributed to differences in the use of adjunctive antithrombotic treatment in the two treatment environments. Interestingly, bleeding complications were more common in the hospital environment where adjunctive antithrombotic treatment was more aggressive, consistent with recent data from RIKS-HIA showing that major bleeding complications among patients receiving fibrinolytic treatment continued to increase from 2001 to 2006 as antithrombotic treatments became more effective. ${ }^{69}$ The availability of potent ADP P2Y12 receptor blockers has raised further concerns about bleeding complications, and it was gratifying, therefore, that the PLATO trial substudy confirmed that event rates could be reduced with ticagrelor compared with clopidogrel without an increase in bleeding risk. ${ }^{7071}$ The role of invasive treatment after fibrinolytic treatment in STEMI has been clarified in two recent meta-analyses of small and medium-size trials comparing strategies of routine early angiography for all patients with deferred or ischaemia-guided angiography. ${ }^{72}$ $\underline{73}$ Both meta-analyses reported that routine early angiography was associated with reductions in the rates of recurrent myocardial infarction and death and this strategy is now recommended in international guidelines.

7.4. Non-ST-segment elevation myocardial infarction

NSTEMI has become the dominant mode of presentation for patients with acute myocardial infarction and in the recent analysis from Kaiser Permanente accounted for $66.9 \%$ of cases. ${ }^{4}$ There has been a perception that NSTEMI is relatively benign despite evidence that prognosis after 2 months becomes substantially worse than with STEMI. 1 ${ }^{74}$ This may explain the tendency of doctors to under-treat NSTEMI based on a mismatch between perceived and actual risk that distorts management decisions, perpetuating the 'treatment-risk paradox'. $\underline{25}$ Thus, despite a worse prognosis, patients with NSTEMI are less likely than patients with STEMI to receive optimal secondary prevention treatment. ${ }^{75}$ Moreover, in a study of 13489 NSTEMI admissions recorded in the MINAP registry, invasive management was associated with better outcomes but was applied inequitably, with lower rates in high-risk groups, including older patients, women and those with cardiac comorbidities. ${ }^{76}$

\subsection{Emergency management}

Dual antiplatelet treatment with aspirin and clopidogrel is central to the management of NSTEMI. ${ }^{77}$ The role of newer more potent ADP P2Y12 receptor blockers remains undetermined, although ticagrelor looks promising, based on its ability to reduce ischaemic events compared with clopidogrel in NSTEMI as well as STEMI, without increasing the risk of bleeding. $\cdot \frac{78}{}$ Simultaneous treatment with fondaparinux is now recommended in preference to enoxaparin, based on the findings in OASIS 5 which compared these agents in 20078 patients with acute coronary syndromes. ${ }^{79}$ Patients randomised to fondaparinux showed a $50 \%$ reduction in major bleeding compared with enoxaparin, with no difference in the incidence of ischaemic events. The reduction in bleeding risk was comparable whether clopidogrel or GPIIb/IIIa receptor blockers were co-prescribed ${ }^{80}$ and cost-effectiveness has now been confirmed. .1 Indications for bivalirudin in NSTEMI have been harder to define and although it has a licence for use in combination with aspirin and clopidogrel, this is based principally on its safety profile (lower bleeding risk), its efficacy for reducing ischaemic events being no greater than either heparin plus GPIIb/IIIa receptor blocker or bivalirudin plus GPIIb/IIIa receptor blockers. $\underline{\text {. }}$

The majority of patients with NSTEMI benefit from interventional management, $\underline{\underline{83}}$ but recent data suggest this could be delayed for at least $24 \mathrm{~h}$ unless continuing clinical instability unresponsive to GPIIb/IIIa receptor blockers calls for earlier action. Thus, in a randomised comparison of immediate versus deferred PCI in 251 patients, the incidence at 30 days of the primary end point, a composite of 
death, non-fatal myocardial infarction or unplanned revascularisation, was significantly higher in the group receiving immediate PCI (60\% vs 39\%). $\underline{\text { s4 }}$ The difference persisted at 6 months' follow-up. Delaying intervention beyond $96 \mathrm{~h}$ is unlikely to be helpful, yet registry data show that this is common, particularly in high-risk patients who have most to gain from revascularisation. $\frac{85}{}$ The evidence for timely revascularisation is largely based on PCI data but a small proportion of patients require coronary artery bypass grafting (CABG). An analysis of US registry data showed that the timing of CABG has no palpable effect on outcomes, the composite of death, myocardial infarction, congestive heart failure, or cardiogenic shock being similar (12.6\% vs 12.4\%) whether CABG is done within $48 \mathrm{~h}$ of admission or later. ${ }^{86}$ In general, therefore, early surgery is recommended to limit hospital stay and reduce resource use.

\section{SECONDARY PREVENTION}

\subsection{Cardiac rehabilitation}

The benefit of cardiac rehabilitation among $30 \quad 161$ Medicare beneficiaries, $20.5 \%$ of whom had recent myocardial infarction, was confirmed by a strong dose-response relationship between the number of rehabilitation sessions attended and long-term rates of death and myocardial infarction. $\frac{87}{}$ Yet a contemporary report of cardiac rehabilitation in the UK found that only $26 \%$ of eligible patients with myocardial infarction are recruited, with adherence

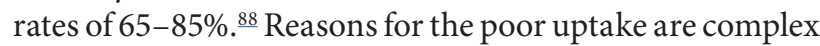
but include the fact that many patients do not want to participate in centre-based group programmes. A systematic review has now reported that home-based programmes are equally effective in improving clinical and health-related quality-of-life outcomes and are more acceptable to many patients. $\stackrel{89}{ }$ Healthcare costs are similar, supporting the further provision of home-based cardiac rehabilitation such as that described by investigators in Birmingham. $\stackrel{90}{\text { The recent }}$ demonstration of improved myocardial blood flow plus reductions in circulating angiogenic cytokines in patients undergoing cardiac rehabilitation provides some reassurance that clinical improvement is physiologically based. .1

\subsection{Lifestyle modification}

An important component of cardiac rehabilitation is lifestyle adjustment to help protect against further coronary events. Top of the list is smoking cessation. A recent study of 1581 patients followed up for 13 years showed that the adjusted HR for all-cause mortality was lower by $43 \%$ in lifelong non-smokers and by $43 \%$ in patients who quit after myocardial infarction. ${ }^{92}$ A new finding was that among persistent smokers, each reduction of five cigarettes smoked per day reduced the risk of death by $18 \%$, providing some comfort for those patients for whom complete abstinence proves impossible. Even among patients who mange to quit, there remains the hazard of second-hand smoke exposure, as reflected by data from Scotland showing that adjusted all-cause and cardiovascular mortality among never-smoking survivors of myocardial infarction increases according to smoke exposure measured by serum cotinine concentration. ${ }^{93}$ The message is clear that protection against recurrent events in survivors of myocardial infarction requires smoking cessation by the patient and also by those with whom the patient makes contact, particularly family members. Together with smoking cessation, advice about exercise and diet delivered in formal programmes can have a salutary effect on modifiable risk profiles, including serum cholesterol, blood pressure and body mass index. ${ }^{94}$ Dietary recommendations usually include $\mathrm{w}-3$ fatty acid supplements ${ }^{95}$ but this has now been questioned by the findings of two studies. In the first, 4837 patients with previous myocardial infarction were randomised to margarines containing marine $\mathrm{n}-3$ fatty acids and plant-derived alinolenic acid in a $2 \times 2$ factorial design. $\underline{96}$ The rate of adverse cardiovascular events did not differ significantly among the study groups. In the second study, highly purified w-3 fatty acids were randomly allocated to 3851 patients with acute myocardial infarction followed up for 12 months. ${ }^{97}$ There were no significant differences in rates of sudden cardiac death (1.5\% vs $1.5 \%)$, total mortality ( $4.6 \%$ vs $3.7 \%$ ), or major adverse cerebrovascular and cardiovascular events (10.4\% vs $8.8 \%$ ) between treatment and placebo groups. The results of these two trials make recommendations for secondary prevention with w-3 fatty acid supplements after myocardial infarction difficult to sustain.

\subsection{Pharmacotherapy}

The importance of optimal secondary prevention after myocardial infarction was emphasised in a modelling study, in which greater absolute gains in survival were achieved by optimising secondary prevention treatments compared with in-hospital reperfusion treatments (104 vs $\leq 30$ lives $/ 10$ 000). $\stackrel{98}{ }$ Recommended are aspirin, b blockers, statins, renin-angiotensin system blockers and thienopyridines - a study of 5353 patients showing that treatment with all five drugs reduced 1-year mortality by $74 \%$ compared with treatment with one or none of them, with consistent effects in STEMI and NSTEMI. $\underline{75}$ Evidence that statins and clopidogrel provide the greatest independent pharmacological benefit (ORs for death 0.85 (0.73 to 0.99 ) and 0.84 (0.72 to 0.99$)$ ) was provided by the GRACE investigators in a nested case-control study of 5148 patients with acute coronary syndromes, $\underline{99}$ and two separate studies have now reported the adverse consequences of failing to adhere to treatment with these drugs during the first year after discharge..$^{100101}$ The message is clear that prescribing secondary prevention treatment according to guideline recommendations and promoting adherence to treatment can together produce further mortality reductions in patients with myocardial infarction.

8.4. Implantable cardioverter-defibrillators (ICDs)

Left ventricular ejection fraction (LVEF) after acute myocardial infarction remains predictive of sudden death in the primary PCI era ${ }^{102}$ and is the key determinant of which patients should be offered an ICD for primary prevention. ${ }^{103}$ However, LVEF in the acute phase is an unreliable guide to LVEF at 3 months when significant recovery of contractile function has often occurred. But there is another reason for delaying decisions about ICDs beyond the guidelinerecommended 40 days. Thus a recent randomised trial of ICD therapy in 898 patients with $\mathrm{LVEF} \leq 40 \%$, recruited within 31 days of acute myocardial infarction, showed no overall mortality reduction for the patients who received an ICD because a high rate of non-sudden death negated 
protection against sudden arrhythmic death provided by the ICD. ${ }^{104}$ A secondary analysis of DINAMIT has now confirmed a high risk of non-sudden death in patients who receive ICDs early after myocardial infarction, while the VALIANT investigators have reported that recurrent infarction or cardiac rupture are common causes of death during this period. 105106 Taken together, these findings explain why ICDs fail to protect against death if implanted early after myocardial infarction. Decisions should, therefore, be deferred, and patients selected for ICD therapy according to measurement of LVEF at 40 days.

\section{CONCLUSION}

The management of acute coronary syndromes continues to evolve and improve. The challenge for cardiovascular researchers is to maintain this momentum and to ensure that the improvements in outcome seen in the developed world have a global impact.

\section{REFERENCES}

1. Mirzaei M, Truswell AS, Taylor R, et al. Coronary heart disease epidemics: not all the same. Heart 2009;95:740-6.

2. Mackay DF, Irfan MO, Haw S, et al. Meta-analysis of the effect of comprehensive smoke-free legislation on acute coronary events. Heart 2010;96:1525-30.

3. Björck L, Rosengren A, Wallentin L, et al. Smoking in relation to ST-segment elevation acute myocardial infarction: findings from the Register of Information and Knowledge about Swedish Heart Intensive Care Admissions. Heart 2009;95:1006-11.

4. Yeh RW, Sidney S, Chandra M, et al. Population trends in the incidence and outcomes of acute myocardial infarction. N Engl J Med 2010;362:2155-65.

5. Myerson M, Coady S, Taylor H, et al. Declining severity of myocardial infarction from 1987 to 2002: the Atherosclerosis Risk in Communities (ARIC) Study. Circulation 2009;119:503-14.

6. Murphy SA, Cannon CP, Wiviott SD, et al. Reduction in recurrent cardiovascular events with intensive lipid-lowering statin therapy compared with moderate lipid-lowering statin therapy after acute coronary syndromes from the PROVE IT-TIMI 22 (Pravastatin or Atorvastatin Evaluation and Infection Therapy-Thrombolysis In Myocardial Infarction 22) trial. J Am Coll Cardiol 2009;54:2358-62.

7. Stone GW, Maehara A, Lansky AJ, et al; PROSPECT Investigators. A prospective natural-history study of coronary atherosclerosis. N Engl J Med 2011;364:226-35.

8. van Velzen JE, Schuijf JD, de Graaf FR, et al. Plaque type and composition as evaluated non-invasively by MSCT angiography and invasively by VH IVUS in relation to the degree of stenosis. Heart 2009;95:1990-6.

9. Hall AS, Barth JH. Universal definition of myocardial infarction. Heart 2009;95:247-9.

10. Reichlin T, Hochholzer W, Bassetti S, et al. Early diagnosis of myocardial infarction with sensitive cardiac troponin assays. N Engl J Med 2009;361:858-67.

11. Mills NL, Churchhouse AM, Lee KK, et al. Implementation of a sensitive troponin I assay and risk of recurrent myocardial infarction and death in patients with suspected acute coronary syndrome. JAMA 2011;305:1210-16.

12. Dekker MS, Mosterd A, van 't Hof AW, et al. Novel biochemi- cal markers in suspected acute coronary syndrome: systematic review and critical appraisal. Heart 2010;96:1001-10.

13. Bruins Slot MH, Reitsma JB, Rutten FH, et al. Heart-type fatty acid-binding protein in the early diagnosis of acute myocardial infarction: a systematic review and meta-analysis. Heart 2010;96:1957-63.

14. Goodacre SW, Bradburn M, Cross E, et al; RATPAC Research Team. The Randomised Assessment of Treatment using Panel Assay of Cardiac Markers (RATPAC) trial: a randomised controlled trial of point-of-care cardiac markers in the emergency department. Heart 2011;97:190-6.

15. Collinson P, Goodacre SW, Gaze D, et al; Very Early Diagnosis Of Chest Pain By Point Of Care Testing. Comparison of the diagnostic efficiency of a panel of cardiac biomarkers compared to troponin measurement alone in The Randomised Assessment Of Panel Assay Of Cardiac Markers (RATPAC) Trial. Heart 2011. [Epub ahead of print]

16. Than M, Cullen L, Reid CM, et al. A 2-h diagnostic protocol to assess patients with chest pain symptoms in the Asia-Pacific region (ASPECT): a prospective observational validation study. Lancet 2011;377:1077-84.

17. Jain S, Ting HT, Bell M, et al. Utility of left bundle branch block as a diagnostic criterion for acute myocardial infarction. Am J Cardiol 2011;107:1111-16.

18. De Winter RJ, Verouden NJ, Wellens HJ, et al. A new ECG sign of proximal LAD occlusion. N Engl J Med 2008;359:2071-3.

19. Verouden NJ, Koch KT, Peters RJ, et al. Persistent precordial "hyperacute" T-waves signify proximal left anterior descending artery occlusion. Heart 2009;95:1701-6.

20. Grenne B, Eek C, Sjøli B, et al. Acute coronary occlusion in non-ST-elevation acute coronary syndrome: outcome and early identification by strain echocardiography. Heart 2010;96:1550-6.

21. Champney KP, Frederick PD, Bueno H, et al; NRMI Investigators. The joint contribution of sex, age and type of myocardial infarction on hospital mortality following acute myocardial infarction. Heart 2009;95:895-9.

22. Wong CK, Gao W, Stewart RA, et al. Relationship of QRS duration at baseline and changes over $60 \mathrm{~min}$ after fibrinolysis to 30-day mortality with different locations of ST elevation myocardial infarction: results from the Hirulog and Early Reperfusion or Occlusion-2 trial. Heart 2009;95:276-82.

23. Goyal A, Mehta SR, Díaz R, et al. Differential clinical outcomes associated with hypoglycemia and hyperglycemia in acute myocardial infarction. Circulation 2009;120:2429-37.

24. Yang SW, Zhou YJ, Hu DY, et al; BEAMIS Study Group. Association between admission hypoglycaemia and in-hospital and 3-year mortality in older patients with acute myocardial infarction. Heart 2010;96:1444-50.

25. Yan AT, Yan RT, Huynh T, et al; Canadian Acute Coronary Syndrome Registry 2 Investigators. Understanding physicians' risk stratification of acute coronary syndromes: insights from the Canadian ACS 2 Registry. Arch Intern Med 2009;169:372-8.

26. Jolly SS, Shenkman H, Brieger D, et al; GRACE Investigators. Quantitative troponin and death, cardiogenic shock, cardiac arrest and new heart failure in patients with non-ST-segment elevation acute coronary syndromes (NSTE ACS): insights from the Global Registry of Acute Coronary Events. Heart 2011;97:197-202. 
27. He LP, Tang XY, Ling WH, et al. Early C-reactive protein in the prediction of long-term outcomes after acute coronary syndromes: a meta-analysis of longitudinal studies. Heart 2010;96:339-46.

28. Eggers KM, Lagerqvist B, Venge P, et al. Prognostic value of biomarkers during and after non-ST-segment elevation acute coronary syndrome. J Am Coll Cardiol 2009;54:357-64.

29. Damman P, Beijk MA, Kuijt WJ, et al. Multiple biomarkers at admission significantly improve the prediction of mortality in patients undergoing primary percutaneous coronary intervention for acute ST-segment elevation myocardial infarction. J Am Coll Cardiol 2011;57:29-36.

30. Gale CP, Manda SO, Weston CF, et al. Evaluation of risk scores for risk stratification of acute coronary syndromes in the Myocardial Infarction National Audit Project (MINAP) database. Heart 2009;95:221-7.

31. Meune C, Drexler B, Haaf P, et al. The GRACE score's performance in predicting in-hospital and 1-year outcome in the era of high-sensitivity cardiac troponin assays and B-type natriuretic peptide. Heart 2011;97:1479-83.

32. Myocardial Ischaemia National Audit Project. Tenth public report 2011. www.ucl.ac.uk/nicor/audits/minap.

33. Nielsen PH, Maeng M, Busk M, et al; DANAMI-2 Investigators. Primary angioplasty versus fibrinolysis in acute myocardial infarction: long-term follow-up in the Danish acute myocardial infarction 2 trial. Circulation 2010;121:1484-91.

34. Saia F, Marrozzini C, Ortolani P, et al. Optimisation of therapeutic strategies for ST-segment elevation acute myocardial infarction: the impact of a territorial network on reperfusion therapy and mortality. Heart 2009;95:370-6.

35. Huber K, Goldstein P, Danchin N, et al. Network models for large cities: the European experience. Heart 2010;96:164-9.

36. Lambert L, Brown K, Segal E, et al. Association between timeliness of reperfusion therapy and clinical outcomes in ST-elevation myocardial infarction. JAMA 2010;303:2148-55.

37. Rathore SS, Curtis JP, Chen J, et al; National Cardiovascular Data Registry. Association of door-to-balloon time and mortality in patients admitted to hospital with ST elevation myocardial infarction: national cohort study. BMJ 2009;338:b1807.

38. Terkelsen CJ, Sørensen JT, Maeng M, et al. System delay and mortality among patients with STEMI treated with primary percutaneous coronary intervention. JAMA 2010;304:763-71.

39. Diercks DB, Owen KP, Kontos MC, et al. Gender differences in time to presentation for myocardial infarction before and after a national women's cardiovascular awareness campaign: a temporal analysis from the Can Rapid Risk Stratification of Unstable Angina Patients Suppress ADverse Outcomes with Early Implementation (CRUSADE) and the National Cardiovascular Data Registry Acute Coronary Treatment and Intervention Outcomes Network-Get with the Guidelines (NCDR ACTION Registry-GWTG). Am Heart J 2010;160:80-7.e3.

40. Amoroso G, Kiemeneij F. Transradial access for primary percutaneous coronary intervention: the next standard of care? Heart 2010;96:1341-4.

41. Jolly SS, Yusuf S, Cairns J, et al; RIVAL Trial Group. Radial versus femoral access for coronary angiography and intervention in patients with acute coronary syndromes (RIVAL): a randomised, parallel group, multicentre trial. Lancet 2011;377:1409-20

42. Hetherington SL, Adam Z, Morley R, et al. Primary percuta- neous coronary intervention for acute ST-segment elevation myocardial infarction: changing patterns of vascular access, radial versus femoral artery. Heart 2009;95:1612-18.

43. Patterson T, Foale RA. If the radial artery is the new standard of care in primary percutaneous coronary intervention, why is most intervention done by the femoral approach? Heart 2011;97:521-52.

44. Brueck M, Bandorski D, Kramer W, et al. A randomized comparison of transradial versus transfemoral approach for coronary angiography and angioplasty. JACC Cardiovasc Interv 2009;2:1047-54.

45. Lo TS, Nolan J, Fountzopoulos E, et al. Radial artery anomaly and its influence on transradial coronary procedural outcome. Heart 2009;95:410-15.

46. Stone GW, Witzenbichler B, Guagliumi G, et al; HORIZONSAMI Trial Investigators. Heparin plus a glycoprotein IIb/IIIa inhibitor versus bivalirudin monotherapy and paclitaxel-eluting stents versus bare-metal stents in acute myocardial infarction (HORIZONS-AMI): final 3-year results from a multicentre, randomised controlled trial. Lancet 2011;377:2193-204.

47. Politi L, Sgura F, Rossi R, et al. A randomised trial of targetvessel versus multi-vessel revascularisation in ST-elevation myocardial infarction: major adverse cardiac events during long-term follow-up. Heart 2010;96:662-7.

48. Vlaar PJ, Mahmoud KD, Holmes DR Jr, et al. Culprit vessel only versus multivessel and staged percutaneous coronary intervention for multivessel disease in patients presenting with ST-segment elevation myocardial infarction: a pairwise and network meta-analysis. J Am Coll Cardiol 2011;58:692-703.

49. Kornowski R, Mehran R, Dangas G, et al; HORIZONSAMI Trial Investigators. Prognostic impact of staged versus "one-time" multivessel percutaneous intervention in acute myocardial infarction: analysis from the HORIZONS-AMI (harmonizing outcomes with revascularization and stents in acute myocardial infarction) trial. J Am Coll Cardiol 2011;58:704-11.

50. Svilaas T, Vlaar PJ, van der Horst IC, et al. Thrombus aspiration during primary percutaneous coronary intervention. $\mathrm{N}$ Engl J Med 2008;358:557-67.

51. Vlaar PJ, Svilaas T, van der Horst IC, et al. Cardiac death and reinfarction after 1 year in the Thrombus Aspiration During Percutaneous coronary Intervention in Acute Myocardial Infarction Study (TAPAS): a 1-year follow-up study. Lancet 2008;371:1915-20.

52. Sardella G, Mancone M, Bucciarelli-Ducci C, et al. Thrombus aspiration during primary percutaneous coronary intervention improves myocardial reperfusion and reduces infarct size: the EXPIRA (thrombectomy with export catheter in infarct-related artery during primary percutaneous coronary intervention) prospective, randomized trial. J Am Coll Cardiol 2009;53:309-15.

53. De Vita M, Burzotta F, Porto I, et al. Thrombus aspiration in ST elevation myocardial infarction: comparative efficacy in patients treated early and late after onset of symptoms. Heart 2010;96:1287-90.

54. Migliorini A, Stabile A, Rodriguez AE, et al; JETSTENT Trial Investigators. Comparison of AngioJet rheolytic thrombectomy before direct infarct artery stenting with direct stenting alone in patients with acute myocardial infarction: The JETSTENT Trial. J Am Coll Cardiol 2010;56:1298-306. 
55. Haeck JD, Kuijt WJ, Koch KT, et al. Infarct size and left ventricular function in the PRoximal Embolic Protection in Acute myocardial infarction and Resolution of ST-segment Elevation (PREPARE) trial: ancillary cardiovascular magnetic resonance study. Heart 2010;96:190-5.

56. Burzotta F, De Vita M, Gu YL, et al. Clinical impact of thrombectomy in acute ST-elevation myocardial infarction: an individual patient-data pooled analysis of 11 trials. Eur Heart J 2009;30:2193-203.

57. Eshaghian S, Shah PK, Kaul S. Advances in antiplatelet treatment for acute coronary syndromes. Heart 2010;96:656-61.

58. Montalescot G, Wiviott SD, Braunwald E, et al; TRITONTIMI 38 Investigators. Prasugrel compared with clopidogrel in patients undergoing percutaneous coronary intervention for ST-elevation myocardial infarction (TRITON-TIMI 38): double-blind, randomised controlled trial. Lancet 2009;373:723-31.

59. Cannon CP, Harrington RA, James S, et al; PLATelet Inhibition and Patient Outcomes Investigators. Comparison of ticagrelor with clopidogrel in patients with a planned invasive strategy for acute coronary syndromes (PLATO): A randomised double-blind study. Lancet 2010;375:283-93.

60. Iijima R, Byrne RA, Ndrepepa G, et al. Pre-procedural C-reactive protein levels and clinical outcomes after percutaneous coronary interventions with and without abciximab: pooled analysis of four ISAR trials. Heart 2009;95:107-12.

61. Hansen PR, Iversen A, Abdulla J. Improved clinical outcomes with intracoronary compared to intravenous abciximab in patients with acute coronary syndromes undergoing percutaneous coronary intervention: a systematic review and meta-analysis. J Invasive Cardiol 2010;22:278-82.

62. Akerblom A, James SK, Koutouzis M, et al. Eptifibatide is noninferior to abciximab in primary percutaneous coronary intervention: results from the SCAAR (Swedish Coronary Angiography and Angioplasty Registry). J Am Coll Cardiol 2010;56:470-5.

63. Zeymer U, Margenet A, Haude M, et al. Randomized comparison of eptifibatide versus abciximab in primary percutaneous coronary intervention in patients with acute ST-segment elevation myocardial infarction: results of the EVA-AMI Trial. J Am Coll Cardiol 2010;56:463-9.

64. Smit JJ, van Werkum JW, ten Berg J, et al; Ongoing Tirofiban in Myocardial Infarction Evaluation (On-TIME) Trial Investigators. Prehospital triple antiplatelet therapy in patients with acute ST elevation myocardial infarction leads to better platelet aggregation inhibition and clinical outcome than dual antiplatelet therapy. Heart 2010;96:1815-20.

65. Mehta SR, Boden WE, Eikelboom JW, et al; OASIS 5 and 6 Investigators. Antithrombotic therapy with fondaparinux in relation to interventional management strategy in patients with ST- and non-ST-segment elevation acute coronary syndromes: an individual patient-level combined analysis of the Fifth and Sixth Organization to Assess Strategies in Ischemic Syndromes (OASIS 5 and 6) randomized trials. Circulation 2008;118:2038-46.

66. Stone GW, Witzenbichler B, Guagliumi G, et al; HORIZONSAMI Trial Investigators. Bivalirudin during primary PCI in acute myocardial infarction. N Engl J Med 2008;358:2218-30.

67. Wailoo A, Goodacre S, Sampson F, et al. Primary angioplasty versus thrombolysis for acute ST-elevation myocardial infarc- tion: an economic analysis of the National Infarct Angioplasty project. Heart 2010;96:668-72.

68. Horne S, Weston C, Quinn T, et al. The impact of pre-hospital thrombolytic treatment on re-infarction rates: analysis of the Myocardial Infarction National Audit Project (MINAP). Heart 2009;95:559-63.

69. Oldgren J, Wernroth L, Stenestrand U; RIKS-HIA Registry, Sweden. Fibrinolytic therapy and bleeding complications: risk predictors from RIKS-HIA. Heart 2010;96:1451-7.

70. Steg PG, James S, Harrington RA, et al; PLATO Study Group. Ticagrelor versus clopidogrel in patients with ST-elevation acute coronary syndromes intended for reperfusion with primary percutaneous coronary intervention: A Platelet Inhibition and Patient Outcomes (PLATO) trial subgroup analysis. Circulation 2010;122:2131-41.

71. James SK, Roe MT, Cannon CP, et al; PLATO Study Group. Ticagrelor versus clopidogrel in patients with acute coronary syndromes intended for non-invasive management: substudy from prospective randomised PLATelet inhibition and patient Outcomes (PLATO) trial. BMJ 2011;342:d3527.

72. Wijeysundera HC, You JJ, Nallamothu BK, et al. An early invasive strategy versus ischaemia-guided management after fibrinolytic therapy for ST-segment elevation myocardial infarction: a meta-analysis of contemporary randomized controlled trials. Am Heart J 2008;156:564-72.

73. Desch S, Eitel I, Rahimi K, et al. Timing of invasive treatment after fibrinolysis in ST elevation myocardial infarction-a meta-analysis of immediate or early routine versus deferred or ischemia-guided randomised controlled trials. Heart 2010;96:1695-702.

74. Chan MY, Sun JL, Newby LK, et al. Long-term mortality of patients undergoing cardiac catheterization for ST-elevation and non-ST-elevation myocardial infarction. Circulation 2009;119:3110-17.

75. Bramlage $\mathrm{P}$, Messer C, Bitterlich N, et al. The effect of optimal medical therapy on 1-year mortality after acute myocardial infarction. Heart 2010;96:604-9.

76. Birkhead JS, Weston CFM, Chen R. Determinants and outcomes of coronary angiography after non-ST-segment elevation myocardial infarction. A cohort study of the Myocardial Ischaemia National Audit Project (MINAP). Heart 2009;95:1593-9.

77. Gray HH, Henderson RA, de Belder MA, et al; Guideline Development Group. Early management of unstable angina and non-ST-segment elevation myocardial infarction: summary of NICE guidance. Heart 2010;96:1662-8.

78. Wallentin L, Becker RC, Budaj A, et al. Ticagrelor versus clopidogrel in patients with acute coronary syndromes. N Engl J Med 2009;361:1045-57.

79. Yusuf S, Mehta SR, Chrolavicius S, et al. Comparison of fondaparinux and enoxaparin in acute coronary syndromes. N Engl J Med 2006;354:1464-76.

80. Jolly SS, Faxon DP, Fox KA, et al. Efficacy and safety of fondaparinux versus enoxaparin in patients with acute coronary syndromes treated with glycoprotein IIb/IIIa inhibitors or thienopyridines: results from the OASIS 5 (Fifth Organization to Assess Strategies in Ischemic Syndromes) trial. J Am Coll Cardiol 2009;54:468-76.

81. Sculpher MJ, Lozano-Ortega G, Sambrook J, et al. Fondaparinux versus Enoxaparin in non-ST-elevation acute 
coronary syndromes: short-term cost and long-term costeffectiveness using data from the Fifth Organization to Assess Strategies in Acute Ischemic Syndromes Investigators (OASIS-5) trial. Am Heart J 2009;157:845-52.

82. Stone GW, McLaurin BT, Cox DA, et al. Bivalirudin for patients with acute coronary syndromes. N Engl J Med 2006;355:2203-16.

83. O'Donoghue M, Boden WE, Braunwald E, et al. Early invasive vs conservative treatment strategies in women and men with unstable angina and non-ST-segment elevation myocardial infarction: a meta-analysis. JAMA 2008;300:71-80.

84. Riezebos RK, Ronner E, Ter Bals E, et al; OPTIMA trial. Immediate versus deferred coronary angioplasty in nonST-segment elevation acute coronary syndromes. Heart 2009;95:807-12.

85. Swanson N, Montalescot G, Eagle KA, et al; GRACE Investigators. Delay to angiography and outcomes following presentation with high-risk, non-ST-elevation acute coronary syndromes: results from the Global Registry of Acute Coronary Events. Heart 2009;95:211-15.

86. Parikh SV, de Lemos JA, Jessen ME, et al; CRUSADE and ACTION Registry-GWTG Participants. Timing of in-hospital coronary artery bypass graft surgery for non-ST-segment elevation myocardial infarction patients results from the National Cardiovascular Data Registry ACTION RegistryGWTG (Acute Coronary Treatment and Intervention Outcomes Network Registry-Get With The Guidelines). JACC Cardiovasc Interv 2010;3:419-27.

87. Hammill BG, Curtis LH, Schulman KA, et al. Relationship between cardiac rehabilitation and long-term risks of death and myocardial infarction among elderly Medicare beneficiaries. Circulation 2010;121:63-70.

88. Bethell H, Lewin R, Dalal H. Cardiac rehabilitation in the United Kingdom. Heart 2009;95:271-5.

89. Dalal HM, Zawada A, Jolly K, et al. Home based versus centre based cardiac rehabilitation: Cochrane systematic review and meta-analysis. BMJ 2010;340:b5631.

90. Jolly K, Lip GY, Taylor RS, et al. The Birmingham rehabilitation uptake maximisation study (BRUM): a randomised controlled trial comparing home-based with centre-based cardiac rehabilitation. Heart 2009;95:36-42.

91. Lee BC, Hsu HC, Tseng WY, et al. Effect of cardiac rehabilitation on angiogenic cytokines in postinfarction patients. Heart 2009;95:1012-18.

92. Gerber Y, Rosen LJ, Goldbourt U, et al; Israel Study Group on First Acute Myocardial Infarction. Smoking status and longterm survival after first acute myocardial infarction: A population-based cohort study. J Am Coll Cardiol 2009;54:2382-7.

93. Pell JP, Haw S, Cobbe S, et al. Secondhand smoke exposure and survival following acute coronary syndrome: prospective cohort study of 1261 consecutive admissions among neversmokers. Heart 2009;95:1415-18.

94. Redfern J, Briffa T, Ellis E, et al. Choice of secondary prevention improves risk factors after acute coronary syndrome: 1-year follow-up of the CHOICE (Choice of Health Options In prevention of Cardiovascular Events) randomised controlled trial. Heart 2009;95:468-75.

95. Khavandi A, Khavandi K, Greenstein A, et al. n-3 Polyunsaturated fatty acids are still underappreciated and underused post myocardial infarction. Heart 2009;95:540-1.

96. Kromhout D, Giltay EJ, Geleijnse JM; Alpha Omega Trial Group. n-3 fatty acids and cardiovascular events after myocardial infarction. N Engl J Med 2010;363:2015-26.

97. Rauch B, Schiele R, Schneider S, et al; OMEGA Study Group. OMEGA, a randomized, placebo-controlled trial to test the effect of highly purified omega- 3 fatty acids on top of modern guideline-adjusted therapy after myocardial infarction. Circulation 2010;122:2152-9.

98. Chew DP, Huynh LT, Liew D, et al. Potential survival gains in the treatment of myocardial infarction. Heart 2009;95:184450 .

99. Chew DP, Anderson FA, Avezum A, et al; GRACE Investigators. Six-month survival benefits associated with clinical guideline recommendations in acute coronary syndromes. Heart 2010;96:1201-6.

100. Daskalopoulou SS, Delaney JA, Filion KB, et al. Discontinuation of statin therapy following an acute myocardial infarction: a population-based study. Eur Heart J 2008;29:2083-91.

101. Boggon R, van Staa TP, Timmis A, et al. Clopidogrel discontinuation after acute coronary syndromes: frequency, predictors and associations with death and myocardial infarction. A hospital registry-primary care linked cohort (MINAPGPRD). Eur Heart J. 2011. [Epub ahead of print]

102. Shiga T, Hagiwara N, Ogawa H, et al; Heart Institute of Japan Acute Myocardial Infarction-II (HIJAMI-II) Investigators. Sudden cardiac death and left ventricular ejection fraction during long-term follow-up after acute myocardial infarction in the primary percutaneous coronary intervention era: results from the HIJAMI-II registry. Heart 2009;95:216-20.

103. Liew R. Prediction of sudden arrhythmic death following acute myocardial infarction. Heart 2010;96:1086-94.

104. Steinbeck G, Andresen D, Seidl K, et al. Defibrillator implantation early after myocardial infarction. N Engl J Med 2009;361:1427-36.

105. Dorian P, Hohnloser SH, Thorpe KE, et al. Mechanisms underlying the lack of effect of implantable cardioverterdefibrillator therapy on mortality in high-risk patients with recent myocardial infarction: insights from the Defibrillation in Acute Myocardial Infarction Trial (DINAMIT). Circulation 2010;122:2645-52.

106. Pouleur AC, Barkoudah E, Uno H, et al; VALIANT Investigators. Pathogenesis of sudden unexpected death in a clinical trial of patients with myocardial infarction and left ventricular dysfunction, heart failure, or both. Circulation 2010;122:597-602.

Corresponding author: prof Andrew L Clark, MD. British Society for Heart Failure, London, UK.E-mail: a.I.clark@hull.ac.uk 